\title{
(C. COBENCE

\section{O FISHBOWL COMO ESTRATÉGIA DE DISCUSSÃO E APRENDIZAGEM ATIVA NO ENSINO PRESENCIAL E ON-LINE}

DOI: 10.37702/2175-957X.COBENGE.2021.3545

Flávio Yukio Watanabe - fywatanabe@ufscar.br

Universidade Federal de São Carlos

Rod. Washington Luís km 235

13565-905 - São Carlos - SP

Ana Carolina Sartorato Beleza - acbeleza@gmail.com

UFSCar

Rua Ray Wesley Herrick 1501

55169-816 - São Carlos - SP

Andrea Aparecida Contini - apcontini@ufscar.br

UFSCAR

Rua Itapura 202

14090-082 - Ribeirão Preto - SP

Anielle Cristhine de Medeiros Takahashi - anielletakahashi@gmail.com

Ufscar

Av Pedro muszkat 1000

13565-543 - São Carlos - SP

Armando Ítalo Sette Antonialli - antonialli@ufscar.br

UFSCar

Rod. Washington Luís, km 235

13565-905 - São Carlos - SP

Ester Almeida Helmer - ester.helmer@ufscar.br

UNIVERSIDADE FEDERAL DE SÃO CARLOS

RUA DA AGROENERGIA 123

13566-421 - SÃO CARLOS - SP

Flávia Gomes Pileggi Gonçalves - flaviapileggi@ufscar.br Universidade Federal de São Carlos

Rua São José 2970

14025-186 - Ribeirão Preto - SP 
Karina Gramani Say - kagramanis@yahoo.com.br

Universidade Federal de São Carlos

Rua São José 409

14010-160 - Ribeirão Preto - SP

Roberta de Fátima Carreira Moreira Padovez - roberta.carreira@gmail.com

Universidade Federal de São Carlos

Rua Ray Wesley Herrick 1501

13565-090 - São Carlos - SP

Resumo: As metodologias ativas de ensino-aprendizagem na educação superior não são exatamente uma novidade, mas sua valorização e consolidação em cursos de engenharia é algo relativamente recente, especialmente no Brasil. Sua eficácia em relação a estratégias consolidadas e tradicionais como aulas expositivas e de laboratório é sempre uma preocupação, mas estudos indicam que as estratégias de aprendizagem ativa podem trazer benefícios, por exemplo, desempenho acadêmico mais elevado e promoção da aprendizagem significativa. Dentre as diferentes estratégias ativas, o Fishbowl (ou Aquário em português) é uma estratégia diferenciada de discussão que promove a escuta ativa, participação organizada e debate de ideias ou pontos de vista distintos. O formato básico de uma sessão de Fishbowl é o de cadeiras organizadas em círculos concêntricos, sendo que a discussão se concentra no círculo interno, o aquário, mas que pode ser acessado de forma ordenada pelos outros participantes dos círculos externos, dando a oportunidade de todos contribuírem com a discussão. Na engenharia, pode ser utilizado para discutir projetos, situações-problema, ou temas de caráter mais geral como questões ambientais, relações étnico-raciais, direitos humanos e desenho universal. Embora o Fishbowl tenha sido concebido para ocorrer de forma presencial, existem alternativas que possibilitam sua implantação de forma semipresencial ou de forma totalmente remota, por meio de ambientes virtuais de comunicação e interação. Além das vantagens enquanto estratégia de discussão e aprendizagem, o Fishbowl potencializa o desenvolvimento do pensamento crítico-reflexivo e estimula o incremento de habilidades, comportamentos e atitudes essenciais ao bom exercício profissional dos futuros engenheiros.

Palavras-chave: Metodologias ativas. Aquário. Debate. Brainstorming. Dinâmica de grupo. 


\section{O FISHBOWL COMO ESTRATÉGIA DE DISCUSSÃO E APRENDIZAGEM ATIVA NO ENSINO PRESENCIAL E ON-LINE}

\section{INTRODUÇÃO}

Atualmente, as metodologias ativas de ensino-aprendizagem na educação superior encontram-se em um processo de valorização e consolidação, especialmente em algumas áreas de formação como as identificadas por STEM, sigla em inglês para "Science, Technology, Engineering and Mathematics", e isso pode ser observado nas novas Diretrizes Curriculares Nacionais do Curso de Graduação em Engenharia, estabelecidas pela Resolução № 2, de 24 de Abril de 2019 (BRASIL, 2019). Entretanto, conforme relatado por Bonwell e Eison (1991), até algumas décadas atrás, não havia um entendimento claro do que seria aprendizagem ativa, uma vez que algumas correntes educacionais defendiam que não seria possível alguém aprender de forma passiva e que todo processo de aprendizagem era ativo por natureza.

Ryan e Martens (1989) contribuíram nesta discussão, apontando que os estudantes aprendem tanto passivamente quanto ativamente, sendo que a aprendizagem passiva ocorre quando os estudantes assumem o papel de "receptáculos do conhecimento", ou seja, eles não participam diretamente no processo de aprendizagem, e o exemplo mais notório é o de aulas expositivas; por sua vez, a aprendizagem ativa tem maior probabilidade de ocorrer quando os estudantes estão fazendo algo que vá além de ouvir, por exemplo questionando e discutindo os temas abordados, resolvendo problemas, realizando experimentos, elaborando projetos, analisando e solucionando problemas ou realizando atividades de campo. Nesse contexto, Bonwell e Eison (1991) indicaram que uma estratégia de aprendizagem ativa pode ser definida como qualquer atividade instrucional com o envolvimento efetivo dos estudantes, realizando coisas e pensando sobre o que estão executando, com o objetivo de atingir uma aprendizagem significativa.

A preocupação com a eficácia das metodologias ativas no aprendizado é um tema presente em diversas pesquisas e neste sentido, Freeman et al. (2014) conduziram uma extensa metanálise de 225 estudos buscando identificar possíveis diferenças no desempenho de estudantes em cursos das áreas de STEM que empregam aulas expositivas tradicionais $(n=67)$ ou aprendizagem ativa $(n=158)$. Dados sobre notas de exames e taxas de reprovação foram examinados e os resultados indicaram que a pontuação média nos exames melhorou cerca de $6 \%$ quando foram adotadas atividades com estratégias de aprendizagem ativa e que os estudantes de aulas tradicionais tinham 1,5 vezes mais probabilidade de reprovação que os alunos em turmas com aprendizagem ativa.

Prince (2004) também analisou a efetividade das estratégias ativas de aprendizagem e realizou uma revisão sobre o tema, abordando diferentes estratégias discutidas e empregadas usualmente em cursos de engenharia. Além da questão da aprendizagem, foram evidenciados benefícios em relação a aspectos atitudinais dos estudantes, bem como na forma de raciocínio e na comunicação. Entretanto, o autor indica que a aprendizagem 
ativa não é a "cura" para todos os problemas educacionais, mas que o corpo docente de engenharia deve estar ciente desses diferentes métodos de ensino e se esforçar para identificar quais se aplicam aos seus objetivos formativos.

Dentre as estratégias ativas de aprendizagem, a discussão em pequenos ou grandes grupos é uma alternativa interessante para se debater questões polêmicas, realizar dinâmicas criativas do tipo brainstorming, promover a escuta atenta e interativa, ou discutir projetos de engenharia. Nesse contexto, o presente trabalho apresenta os fundamentos e os diferentes formatos e contextos de aplicação da estratégia de discussão Fishbowl (Aquário, em português), bem como sua aplicação em atividades educacionais no ensino presencial, semipresencial e, mais recentemente, de forma adaptada ao ensino on-line.

\section{A ESTRATÉGIA FISHBOWL - ORIGENS E FUNDAMENTOS}

O Fishbowl é uma estratégia de apresentação, escuta ativa e discussão. Segundo Fruchter (2006), a criação do Fishbowl foi inspirada nas práticas vivenciadas nas escolas de medicina nas quais os estudantes observam através de janelas de vidro os procedimentos cirúrgicos realizados por especialistas, aprendendo, portanto, por meio da observação do trabalho de seus mentores.

Historicamente, aulas de anatomia e cirurgias do século XIX e início do século XX eram muitas vezes abertas ao público, como ilustrado na Figura $1 \mathrm{a}$, e as salas de cirurgia mais se assemelhavam a salas de teatro, na qual o público compartilhava o mesmo espaço dos profissionais que realizam os procedimentos cirúrgicos. Com o desenvolvimento da área de microbiologia, o público passou a observar as cirurgias em espaços separados por amplas janelas de vidro e, atualmente, com o auxílio da tecnologia, pode acompanhar detalhes dos procedimentos cirúrgicos transmitidos em tempo real para telas digitais, como na Figura $1 b$.

Figura 1 - Acompanhamento de procedimentos cirúrgicos.

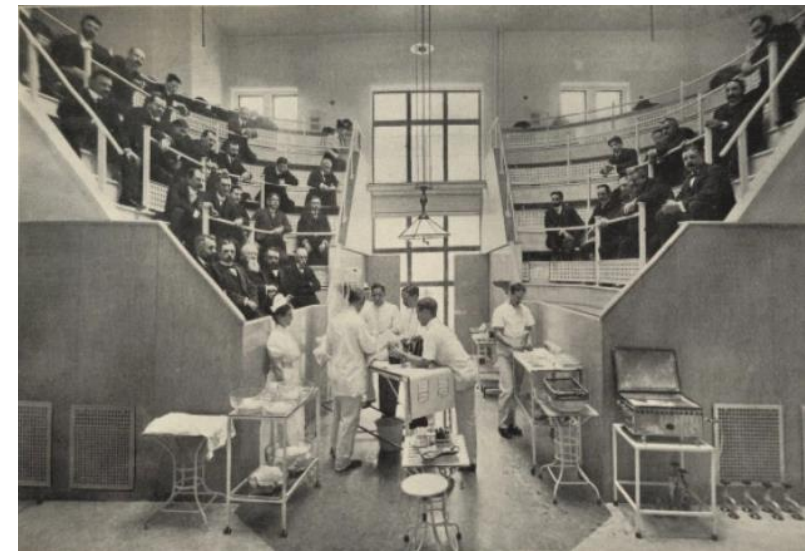

(a) Sala de cirurgia do Presbyterian Hospital (1901) Fonte: Shaner (2014)

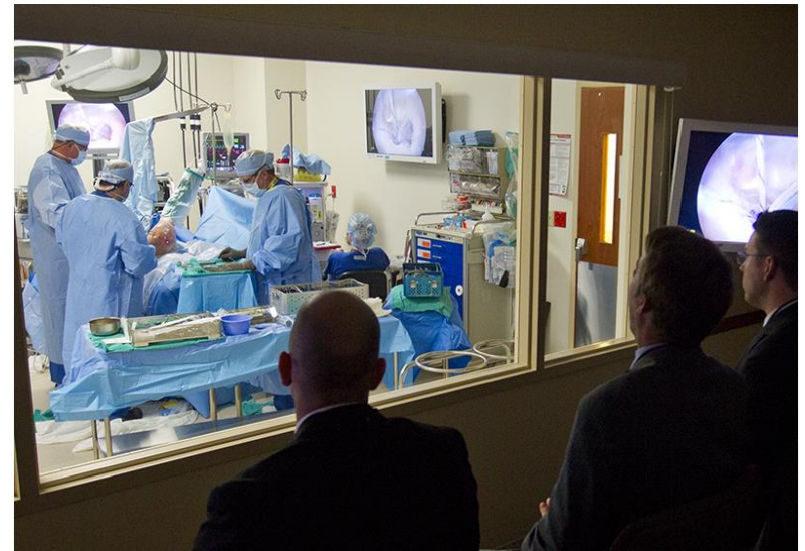

(b) Centro cirúrgico Red Hills - Tallahassee Fonte: Dehart (2013)

Nos cenários apresentados, em princípio, os diálogos ocorrem apenas entre os integrantes dos grupos distintos, separados e diferenciados em especialistas e espectadores, sendo que o aprendizado destes últimos ocorre apenas por meio da observação dos procedimentos e intervenções dos especialistas.

A estratégia Fishbowl foi estruturada inicialmente com o objetivo de reproduzir essa dinâmica, constituindo-se em um método para organizar apresentações e discussões entre um pequeno grupo de especialistas que, de certa forma, expõe um grande grupo de espectadores aos seus conhecimentos, ao mesmo tempo em que expande a compreensão 
coletiva de um determinado assunto. Essa estratégia de discussão e de instrução foi adotada em ambientes empresariais organizacionais, governamentais ou educacionais, e suas principais características são relatadas a seguir com base nas indicações de Eitington (2002), United Nations (2011), Unicef (2015) e Brouwer e Brouwers (2017).

Usado originalmente em conferências, workshops e reuniões, o Fishbowl surgiu como uma alternativa a debates tradicionais ou painéis de discussão e oferece um ambiente mais dinâmico para discutir propostas ou questões polêmicas e compartilhar experiências. O arranjo físico básico do Fishbowl é ilustrado na Figura 2, na qual é possível identificar dois conjuntos de cadeiras organizadas nos contornos de dois círculos concêntricos, sendo as cadeiras do círculo central, denominado "aquário", são ocupadas pelos especialistas, enquanto os espectadores, em maior número, ocupam as cadeiras do círculo externo. Se o número de participantes for muito grande, podem ser organizados dois ou mais círculos externos concêntricos.

Figura 2 - Arranjo físico básico da estratégia Fishbowl

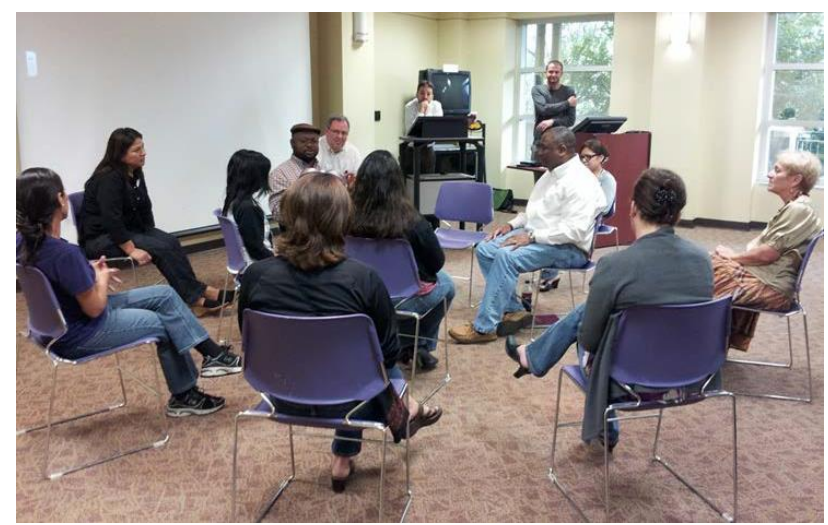

Fonte: Brouwe e Brouwers (2017)

Quando as pessoas do aquário são servidores ou gestores públicos, essa técnica pode ajudar a trazer mais transparência ao processo de deliberação e tomada de decisão, aumentando a confiança e o entendimento sobre as questões debatidas. Além disso, a discussão pode ocorrer como uma "discussão fechada" entre os especialistas do aquário, mas frequentemente a estrutura é modificada para o modo de "discussão aberta", propiciando a interação dos especialistas com os espectadores, mas de forma organizada. Nesse caso, uma ou mais cadeiras do aquário são deixadas vazias, permitindo a participação de "visitantes" que pertencem ao grupo de espectadores, fazendo perguntas, comentários ou apresentando seus pontos de vista.

A discussão deve transcorrer somente entre os participantes que ocupam as cadeiras do aquário, com cerca de 3 a 6 pessoas, sobre questões pré-estabelecidas e sob a supervisão de um moderador. Os demais participantes se tornam ouvintes atentos e participantes em potencial da discussão, além disso, para garantir a oportunidade de participação e a rotatividade de todos os indivíduos, pelo menos uma cadeira do aquário deve ser mantida sempre desocupada.

O diagrama esquemático em vista superior apresentado na Figura 3 ilustra essa dinâmica de trocas de posições no Fishbowl. Inicialmente, conforme indicado na Figura 3a, as cadeiras centrais (azuis) do aquário são ocupadas pelos especialistas, mas deixando pelo menos uma cadeira livre (verde); os demais participantes ocupam as cadeiras do círculo externo (vermelhas) e apenas ouvem atentamente a discussão. Quando algum deles quiser opinar, deve seguir a dinâmica indicada na Figura 3b e ocupar a cadeira livre do aquário (seta (1) e um dos integrantes do aquário deve deixar voluntariamente a 
discussão (seta (2), mantendo sempre uma cadeira livre para que seja possível que outros participantes entrem na discussão. Tanto a sequência das falas quanto a entrada e saída dos participantes no aquário pode ocorrer sem a interferência do moderador, preferencialmente, tornando a atividade um exercício coletivo e autogerido de equilíbrio entre momentos de falas e audição atenta.

Figura 3 - Estrutura básica e funcionamento do Fishbowl

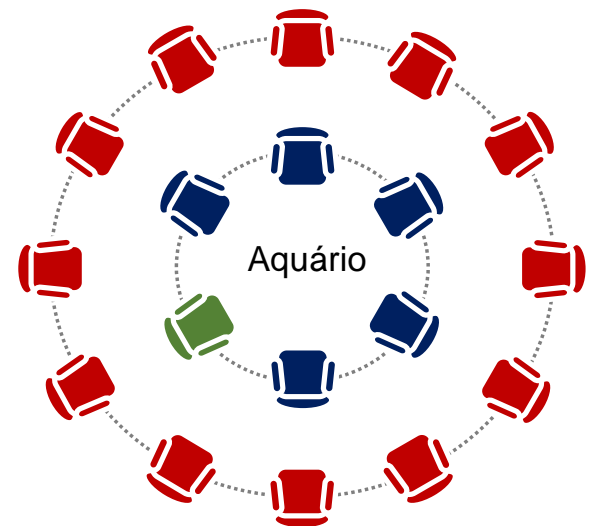

(a) Configuração inicial

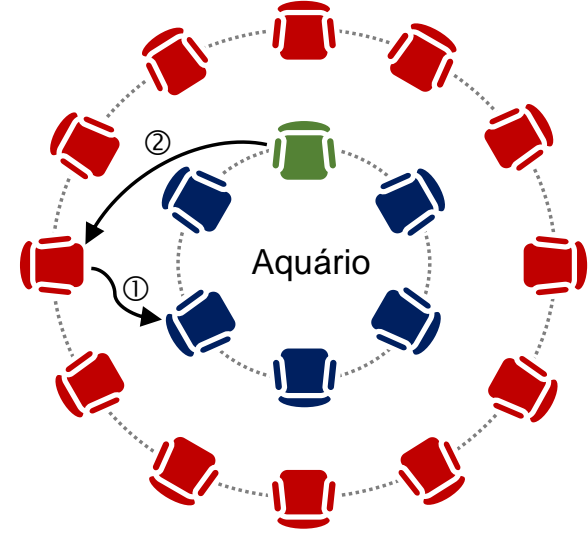

(b) Troca de posições

Fonte: Elaborada pelos autores

As recomendações gerais indicam que o Fishbow/ pode ser empregado para grupos de 12 a 30 pessoas e que as discussões devem durar de 45 a 90 minutos. Recomenda-se também que um dos participantes atue como relator das discussões; entretanto, essas regras não são absolutas e podem ser ajustadas aos contextos e ambientes particulares. $A$ utilização de recursos como o flipcharts, quadros ou blocos de anotação pode ser muito útil para se registrar questões-chave da discussão, facilitando também no processo de fechamento da atividade, no qual o relator apresenta uma síntese com os pontos principais da discussão e as conclusões ou decisões resultantes. A atividade pode prever também a presença de um observador que apresenta suas impressões sobre a dinâmica de participação e interação do grupo, ao final da sessão.

\section{$3 \quad$ O FISHBOWL EM DIFERENTES CONTEXTOS E FORMATOS}

A estratégia Fishbowl pode ser empregada não somente na presença de um grupo de especialistas, mas também em discussões nas quais todos os participantes possuem o mesmo nível de entendimento sobre determinado assunto, como em um grupo de estudantes. Além disso, as regras não são rígidas e podem ser adaptadas aos diferentes contextos de discussão de projetos, planos de ação ou temas polêmicos, seja em ambientes presenciais, semipresenciais ou, mais recentemente, virtuais.

Um ponto em comum é que, em ambientes educacionais, a temática abordada deve ser informada previamente e materiais ou informações podem ser fornecidas ou preparadas previamente para que as discussões sejam mais aprofundadas ou aplicadas, o que caracteriza no campo da educação um procedimento típico da estratégia de Sala de Aula Invertida.

Quanto ao tamanho dos grupos nos quais é possível aplicar o Fishbowl, não há um consenso, mas diferentes formatos possibilitam trabalhar tanto com grupos menores quanto maiores, por meio da composição de subgrupos. 


\subsection{Quando e por que aplicar?}

A estratégia Fishbowl pode ser empregada em diferentes situações e com objetivos diversificados, como evidenciado por Eitington (2002) e a Unicef (2015).

\section{Dinâmica de grupo com a participação ativa de muitas pessoas}

Envolver muitos participantes em uma discussão é um grande desafio, mas a dinâmica de mudanças de posições e a autogestão do grupo nas participações ativas, mantêm os participantes concentrados na discussão em plena evolução, bem como os faz imaginar quem virá a seguir e o que será dito por esta pessoa, mantendo os níveis de atenção e energia elevados.

\section{Introdução e discussão de tópicos controversos.}

A discussão deve ser iniciada com pessoas que possuam um bom entendimento sobre o tópico abordado e tenham pontos de vista diversos, mas também que consideram os prós e contras de forma ampla. Os expectadores serão capazes de ver as várias facetas e pontos de vista emergirem da discussão, o que facilita uma exploração mais objetiva e aprofundada do tópico abordado e seus desdobramentos.

\section{Apresentação de painéis de discussão por especialistas}

A atividade começa com um painel de discussão entre especialistas ou outros participantes convidados, sob a condução do moderador. Enquanto o painel discute o assunto em questão, os expectadores participam ouvindo ativamente. A cadeira vazia colocada no Aquário permite que qualquer ouvinte possa fazer uma breve participação, fazendo uma pergunta ou contribuindo com uma ideia.

\section{Alternativa a mesas redondas}

Usualmente, em uma mesa redonda de discussão participam um mediador e de dois a quatro convidados que compartilham seus conhecimentos sobre um determinado tema por meio de apresentações digitais em telas de projeção. Essas apresentações consecutivas, por mais breves que sejam, podem levar à redução no nível de atenção dos demais participantes e as discussões acabam ficando "descoladas" no fim. Nesse sentido, no Fishbowl as apresentações formais são substituídas por diálogos, garantindo um ritmo mais dinâmico, informal e atrativo.

\section{Treinamento de pequenos grupos}

Atividades de treinamento em ambientes empresariais ou acadêmicos que envolvem momento "quebra-gelo", brainstorming, discussão e solução de problemas e projetos, discussão de pontos de vista divergentes, melhoria das relações interpessoais e de comunicação, identificação dos objetivos da equipe, avaliações de progresso e efetividade de um treinamento.

\subsection{Como aplicar?}

Para a organização de uma sessão de Fishbowl é indicado que sejam seguidas algumas etapas gerais, mas que podem ser adaptadas aos diferentes contextos e objetivos.

\section{Definição prévia do tema}

Praticamente qualquer tema pode ser discutido por meio do Fishbowl, mas os temas mais adequados são aqueles que não possuem uma única resposta ou solução, ou que possuem diferentes interpretações, permitindo um debate de opiniões e visões distintas. Dependendo da aplicação, pode ser indicado aos participantes que realizem leituras ou 
assistam vídeos previamente, como forma de preparação para as discussões. Além disso, se for definido que a discussão contará com a participação de especialistas, estes devem ser informados previamente sobre a dinâmica de funcionamento do Fishbowl.

\section{Organização da sala}

As cadeiras devem ser organizadas em círculos concêntricos, garantindo um espaço suficiente para a movimentação dos participantes. Não existe um número específico de cadeiras que deve compor o círculo central, ou aquário, mas este deve ser definido em função da estratégia adotada considerando a participação ou não de especialistas, o número total de participantes e o número de cadeiras que devem ficar desocupadas simultaneamente no aquário. Se houver a necessidade, locais para anotações com quadros ou flipcharts podem ser disponibilizados aos participantes.

\section{Apresentação do tema e da forma de discussão}

O tema a ser discutido deve ser apresentado ou relembrado e uma visão geral sobre a forma de funcionamento da discussão deve ser esclarecida e discutida com os participantes, visando a menor interferência possível do moderador, uma vez que a dinâmica de participação voluntária na discussão e a ordem das participações devem ser autogeridas pelo grupo, buscando desenvolver aspectos comportamentais como empatia, respeito a opiniões divergentes, equilíbrio entre momentos de escuta e fala, ou tomada de decisão coletiva. O moderador deverá indicar também se alguns dos participantes atuarão voluntariamente como observador ou relator da sessão de Fishbowl.

\section{Análise final}

Uma vez esgotada a discussão, seja por se chegar a um consenso, ou por se entender que existem pontos de vistas distintos e válidos, o moderador deve pedir aos participantes que reflitam sobre os pontos debatidos e o que eles aprenderam com a discussão. Se foi definido previamente que alguns participantes atuassem como relator ou observador, eles devem expor seus relatos. Também pode ser solicitado que os participantes indiquem sugestões de melhoria para a discussão em outras ocasiões, e ainda pode ser adotado processo de autoavaliação e avaliação interpares na participação e contribuição efetiva na discussão.

\subsection{Variações e adaptações}

\section{Fishbowl com os participantes divididos em dois grupos}

A partir de uma temática previamente estabelecida pelo moderador, os participantes são divididos em dois grupos e cada um deles define de duas a quatro questões para o outro grupo. Os integrantes de um grupo ocupam as cadeiras do círculo central do aquário e discutem as questões propostas a eles, enquanto os integrantes do outro grupo atuam apenas como ouvintes atentos. Quando todos os integrantes do grupo no aquário tiveram a oportunidade de falar, as posições e os papéis são invertidos e o outro grupo discute o segundo conjunto de questões.

\section{Feedback Fishbowl}

Variação que sistematiza a interação entre os participantes dos círculos interno e externo. Os integrantes do aquário discutem o assunto por cerca de 15 minutos e, em seguida, viram as cadeiras para ficar de frente para os participantes que ocupam o círculo externo que apresentam seus comentários e feedbacks; a seguir, os membros do aquário retomam a posição inicial e concluem a discussão incorporando as novas informações e 
comentários. Após a conclusão desta rodada, os dois grupos trocam de posição nos círculos e repete-se o processo de discussão-feedback-conclusão.

\section{Fishbowl homogêneo ou heterogêneo}

A participação de especialistas convidados pode ser planejada de modo a configurar um Fishbowl homogêneo, no qual os convidados possuam opiniões ou experiências semelhantes, visando apresentar aos demais participantes conceitos e evidências coesas que corroborem um determinado ponto de vista, evitando embates nos estágios iniciais da discussão. Contrastando com essa configuração, em um Fishbowl heterogêneo os convidados devem ter pontos de vista contrastantes ou divergentes, mas o moderador deve garantir um debate produtivo e no qual as diferentes opiniões sejam igualmente abordadas.

\section{Fishbowl com grandes grupos}

Quando o número de participantes for muito grande ou houver barreiras de linguagem, a indicação é organizar grupos menores de Fishbowl para discussões iniciais, cada qual com um moderador e um representante voluntário. Após esta etapa, os representantes de cada grupo ocupam as posições no aquário e continuam a discussão, mas já com as impressões trazidas pelos colegas dos pequenos grupos. Ao final, 0 fechamento da discussão pode ser feito no grande grupo ou nos pequenos grupos. Todas as outras regras e recomendações para o Fishbowl se aplicam nos dois momentos distintos.

\section{Fishbowl com dramatização}

Esse formato se aplica também a grandes grupos e há a necessidade de se planejar previamente uma situação-problema na qual são definidos papéis distintos a serem representados no debate de determinado tema ou problema. Os participantes são reunidos em grupos associados a cada um dos papéis e cada grupo passa a discutir e se preparar para representar o papel atribuído, embora apenas um dos membros de cada grupo o desempenhe na dramatização. A dramatização ocorre como um Fishbowl fechado, com os participantes ouvintes na parte externa no aquário. A atividade é finalizada com uma discussão e relato dos pontos principais abordados.

\section{$4 \quad$ FISHBOWL NO AMBIENTE VIRTUAL}

A evolução das Tecnologias Digitais da Informação e Comunicação (TDIC) nas últimas décadas têm propiciado mudanças significativas nas formas das pessoas trabalhar, estudar e aprender e se comunicar. $\mathrm{Na}$ área de educação, as TDIC propiciaram uma evolução significativa no Ensino à Distância e na implementação de metodologias ativas de ensino-aprendizagem. O Fishbowl também foi adaptado ao ambiente virtual, conectando pessoas em locais distintos em atividades semipresenciais ou totalmente remotas.

Renate Fruchter (2006), do Problem Based Learning Laboratory (PBL Lab) da Universidade de Stanford, adaptou o Fishbowl como uma estratégia de mentoria de atividades PBL em projetos da área de Arquitetura, Engenharia e Construção, mas envolvendo estudantes de várias universidades ao redor do mundo, participando presencialmente ou remotamente, mas de forma simultânea, como ilustrado na Figura 4.

A estrutura básica do Fishbowl de Fruchter prevê a divisão dos participantes em 3 zonas: na Zona 1, ou Zona de Ação, atuam os especialistas da indústria que opinam sobre os projetos e interagem com os outros participantes; na Zona 2, ou Zona de Reflexão, os indivíduos analisam e fazem questionamentos acerca das informações discutidas na Zona 1; na Zona 3, ou Zona de Observação, que inclui os estudantes remotos, os participantes 
atuam mais como espectadores atentos, mas raramente interagem com os demais participantes das Zonas de Ação e Reflexão, embora isso seja possível e desejável.

Figura 4 - Estrutura Fishbowl do PBL Lab, Universidade de Stanford.
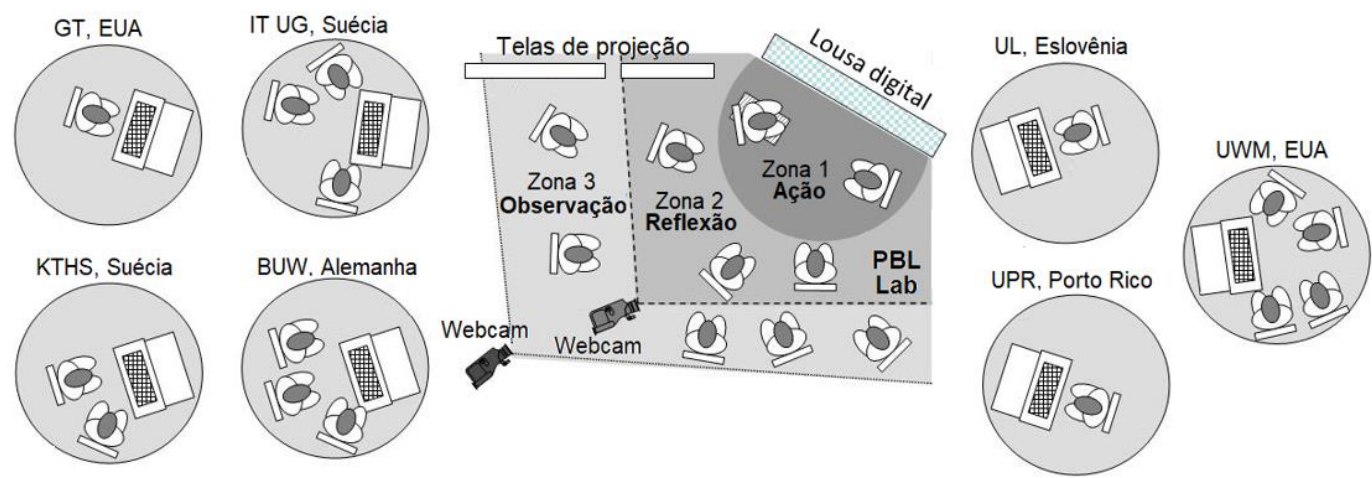

Fonte: Adaptado de Fruchter (2006)

Araújo et al. (2016) adotaram a estratégia de mentoria Fishbowl na Universidade Virtual de São Paulo (UNIVESP), em Projetos Integradores que empregam as estratégias colaborativas Problem and Project Based Learning (PPjBL), com participação presencial ou virtual utilizando o Google Hangouts. Neste caso, equipes de estudantes apresentam e discutem seus projetos com especialistas e, posteriormente, discutem com um orientador pedagógico as contribuições dos especialistas para o desenvolvimento do projeto e produção de um protótipo físico ou digital.

Mais recentemente, no início do ano de 2020, com o agravamento da crise pandêmica de Covid-19 causada pelo coronavírus SARS-CoV-2, o ensino presencial convencional precisou ser adaptado para o ambiente virtual, configurando a modalidade de "ensino remoto emergencial", conforme indicado por Hodges et al. (2020). Outro desafio enfrentado foi adaptar as metodologias de aprendizagem ativa para o ambiente virtual, mas experiências bem-sucedidas como as relatadas por Watanabe et al. (2020) mostraram que isso era possível, especialmente para atividades síncronas.

Propostas de Fishbowl on-line usando diretamente os aplicativos de reuniões como o Google Meet, formatado por Watanabe et al. (2020), e o Zoom Meetings, indicado por Venegas (2020), utilizam os próprios recursos dos aplicativos para se definir quais pessoas estão no aquário ou são ouvintes na discussão. Como nesses aplicativos não é possível dispor as imagens dos participantes em círculos, as pessoas do aquário são identificadas pelo sinal de vídeo ligado, e as demais, com o sinal de vídeo desligado, incluindo relator, observador e moderador, conforme ilustrado na Figura 5a. Sempre que uma pessoa ligar o vídeo, indica que ela "entrou" no aquário e alguém deve "sair" do mesmo desligando o vídeo, mantendo constante o número de pessoas no aquário, como ilustrado na Figura 5b.

Figura 5 - Estrutura do Fishbowl adaptada para um aplicativo de reunião virtual

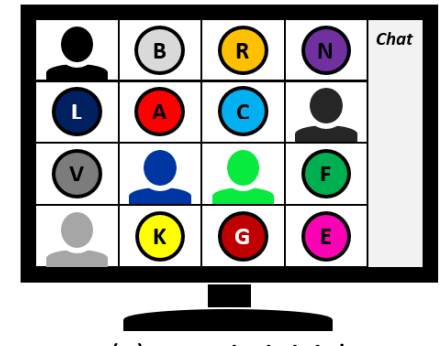

(a) arranjo inicial

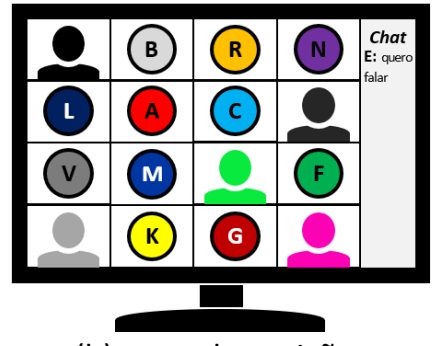

(b) troca de posições

Fonte: Watanabe et al. (2020). 
Embora estas soluções sejam interessantes, a formação tradicional dos círculos do Fishbowl não é preservada. Entretanto, novas alternativas surgiram emulando de certa forma o formato presencial, como o apresentado por Burdett (2020), usando o aplicativo Miro board, ilustrado na Figura 6.

Figura 6 - Fishbowl no ambiente virtual Miro board

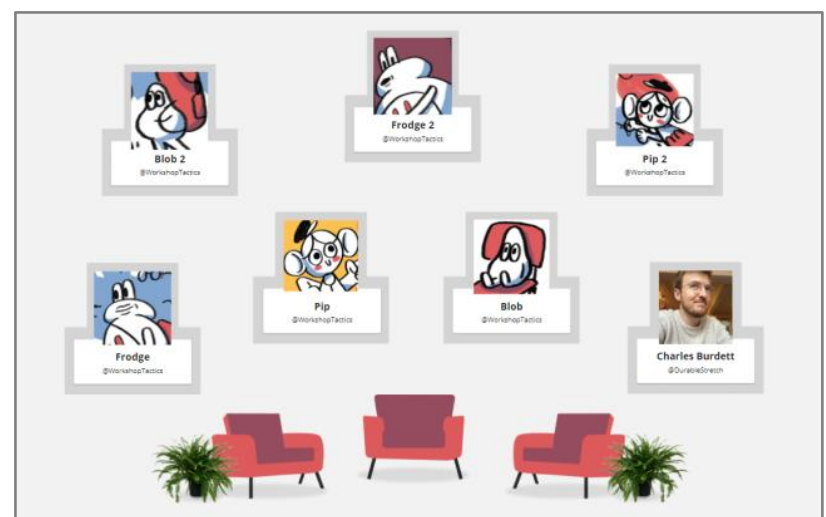

Fonte: Burdett (2020)

Inspirado nessas alternativas, os autores do presente trabalho e que atuam no Grupo de Trabalho em Metodologias Ativas e Estratégias de Avaliação (MetAA) da Universidade Federal de São Carlos (UFSCar), elaboraram um ambiente virtual usando o aplicativo Google Jamboard, ilustrado na Figura 7, que combinado com as ferramentas de conversação do Google Meet, possibilitam uma experiência de discussão muito similar ao Fishbowl presencial.

Figura 7 - Fishbowl no ambiente virtual no Google Jamboad

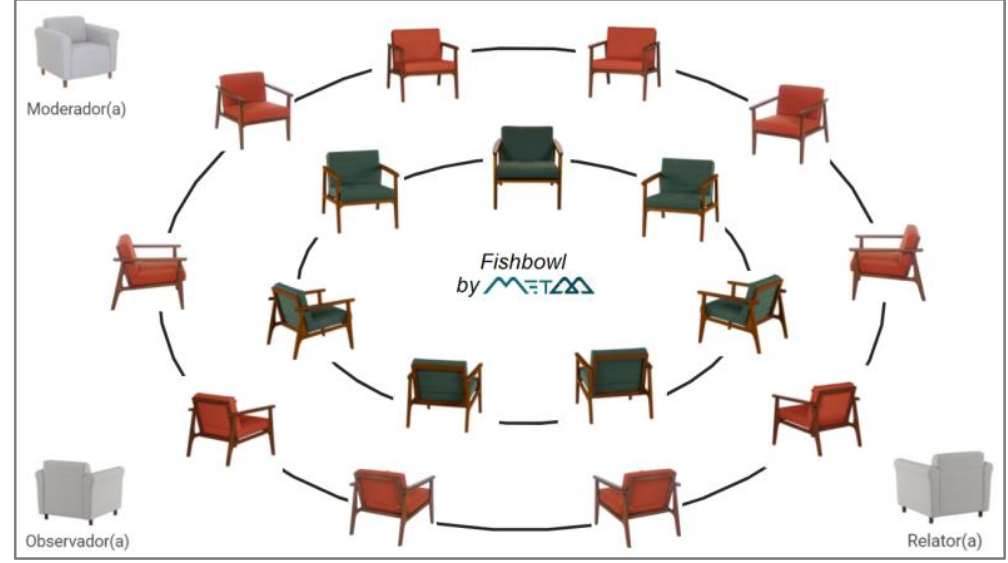

Fonte: Elaborada pelos autores

O número de poltronas nos círculos pode ser previamente ajustado e os participantes podem se identificar por meio de etiquetas ou fotos acompanhadas de seus respectivos nomes. A dinâmica de movimentação das "pessoas" e participação nas discussões ocorrem como em um ambiente presencial do Fishbowl. Um modelo desse tipo de sala foi disponibilizado pelos autores por meio de um hiperlink e pode ser copiado, editado e utilizado, desde de que seja atribuído o crédito de criação ao Grupo MetAA/UFSCar. 


\section{CONSIDERAÇÕES FINAIS}

O Fishbowl constitui uma estratégia de discussão que pode ser adotada em diferentes contextos profissionais e de ensino e com grupos de tamanhos e públicos diversificados, sejam eles heterogêneos ou homogêneos. Aplicações na educação superior são mais frequentes nas áreas de ciências da saúde ou humanas, mas na área de engenharia também são possíveis, por exemplo para discutir projetos e situações-problema de engenharia, ou temas de caráter mais geral como questões ambientais, relações étnicoraciais, direitos humanos e desenho universal.

As principais vantagens da estratégia Fishbowl estão relacionadas às características organizacionais da discussão, como: todos os participantes têm a mesma oportunidade de participar sem ser interrompido e expressando sua opinião sobre o tema debatido; os estudantes podem se tornar ouvintes mais atentos; o pensamento crítico-reflexivo é estimulado e potencializado. O Fishbowl é uma estratégia potente para promover o desenvolvimento da habilidade de comunicação efetiva, muito valorizada nos ambientes profissionais. Por outro lado, por se tratar de uma estratégia na qual as pessoas precisam se expor, algumas delas podem se sentir constrangidas ao ter que manifestar seu ponto de vista e outras podem ter dificuldade em ficar ouvindo sem interromper a fala de outras pessoas.

Para "estimular" que os estudantes participem das discussões em uma atividade Fishbowl, pode-se empregar um processo de autoavaliação e avaliação interpares, indicado previamente aos participantes. Entretanto, entende-se que a vivência nas discussões pode levar a uma formação mais completa dos futuros engenheiros, aprimorando comportamentos e atitudes essenciais ao bom exercício profissional, e isso deve ser enaltecido aos estudantes.

\section{REFERÊNCIAS}

BRASIL. Ministério da Educação. Conselho Nacional de Educação. Câmara de Educação Superior. Resolução no 2, de 24 de abril de 2019. Institui as Diretrizes Curriculares Nacionais do Curso de Graduação em Engenharia. Brasília: Ministério da Educação, 2019. Disponível em: http://portal.mec.gov.br/index.php?option=com docman\&view= download\&alias=112681-rces002-19\&category slug=abril-2019-pdf\&ltemid=30192.

Acesso em 24 de abr. 2021.

BONWELL, C. C.; EISON, J. A. Active Learning: Creating Excitement in the Classroom. Washington, D.C.: The George Washington University, School of Education and Human Development, 1991. (ASHE-ERIC Higher Education Reports Series, n. 1)

BROUWER, H.; BROUWERS, J. The MSP Tool Guide: Sixty tools to facilitate multistakeholder partnerships. Companion to The MSP Guide. Wageningen: Wageningen University and Research, CDI, 2017.

BURDETT, C. How to run a remote virtual Fishbowl discussion. In: Product Club Blog. 30 Jun. 2020. Disponível em: https://product.club/blogs/workshop-strategies/how-to-run-aremote-virtual-fishbowl-discussion. Acesso em: 21 abr. 2021.

DEHART, J. The Red Hills Surgical Center Invites Students to Watch Medical Procedures Up Close and Personal - The Operating Theatre. In: Tallahassee Magazine - Digital

Edition, 2013. Disponível em: https://www.tallahasseemagazine.com/the-red-hillssurgical-center-invites-students-to-watch-medical-procedures-up-close-and-personal/. Acesso em: 18 abr. 2021. 
EITINGTON, J. E. The winning trainer: winning ways to involve people in learning. 4.ed. Woburn: Butterworth-Heinemann, 2002.

FREEMAN, S. et al. Active learning increases student performance in science, engineering, and mathematics. PNAS, v, 111, n. 23, p. 8410-8415, 2014.

FRUCHTER, R. The Fishbowl ${ }^{\mathrm{TM}}$ : Degrees of Engagement in Global Teamwork. In: SMITH, I. F. C. Intelligent Computing in Engineering and Architecture, Berlin-Heidelberg: Springer, 2006, p. 241-257. (EG-ICE Workshop, 13., 2006. Lecture Notes in Artificial Intelligence Series, v. 4200).

HODGES, C. et al. The Difference Between Emergency Remote Teaching and Online Learning. EDUCAUSE Review. 27 mar. 2020. Disponível em: https://er.educause.edu/ articles/2020/3/the-difference-between-emergency-remote-teaching-and-online-learning. Acesso em: 3 jun. 2020.

PRINCE, M. Does Active Learning Work? A Review of the Research. Journal of Engineering Education, v. 93, n. 3, p. 223-231, 2004.

RYAN, M. P.; MARTENS, G. G. Planning a College Course: A Guidebook for the Graduate Teaching Assistant. Ann Arbor, MI: National Center for Research to Improve Postsecondary Teaching and Learning, 1989.

SHANER, A. It's All in the Details. In: Books, Health and History - New York Academy of Medicine, 2014. Disponível em: https://nyamcenterforhistory.org/2014/08/). Acesso em 18 abr. 2021.

UNICEF. Knowledge Exchange Toolbox: Group methods for sharing, discovery and cocreation. New York: UNICEF - Division of Data, Policy and Research, 2015.

UNITED NATIONS. Office of the High Commissioner for Human Rights (OHCHR). Share, learn, innovate! Methods and technologies to share human rights knowledge and ideas. New York/Geneva, 2011.

VENEGAS, B. C. How to do a fishbowl activity in the virtual space? In: Barbara CV Blog. 24 Aug. 2020. Disponível em: https://www.barbaracv.com/blog/a-virtual-fishbowl/. Acesso em: Acesso em: 21 abr. 2021.

WATANABE, F. Y. et al. Formação Docente em Metodologias Ativas e o uso de Tecnologias Digitais de Informação e Comunicação (TDIC) no Ensino Remoto Emergencial. In: Congresso Internacional de Educação e Tecnologias/Encontro de Pesquisadores em Educação a Distância - CIET:EnPED, 5; 2020, São Carlos. Anais [...]. São Carlos: UFSCar, 2020. Disponível em: https://cietenped.ufscar.br/submissao/index.php/2020/article/view/1810/1444. Acesso em: 21 abr. 2021.

\title{
FISHBOWL AS A DISCUSSION STRATEGY AND ACTIVE LEARNING IN CLASSROOM AND ONLINE TEACHING
}

\begin{abstract}
Active teaching and learning methodologies in higher education are not exactly a novelty, but its valorization and consolidation in engineering courses is a relatively recent event, especially in Brazil. Its effectiveness in relation to consolidated and traditional strategies such as lectures and laboratory classes is always a concern, but studies indicate that active learning strategies can bring benefits in terms of higher academic performance
\end{abstract}


and the promotion of meaningful learning. Among the different active strategies, the Fishbowl is a differentiated discussion strategy that promotes active listening, organized participation and debate of different ideas or points of view. The basic format of a Fishbowl session is of chairs arranged in concentric circles, the discussion being concentrated in the inner circle, the aquarium, but which can be accessed in an orderly manner by the other participants in the outer circles, giving everyone the opportunity contribute to the discussion. In engineering, it can be used to discuss projects, problem situations, or more general themes such as environmental issues, ethnic-racial relations, human rights, and universal design. Although the Fishbowl has been designed to take place in person, there are alternatives that allow its implementation in a semi-presential way or in a totally remote way, through virtual environments of communication and interaction. Besides the advantages as a strategy of discussion and learning, the Fishbowl enhances the development of critical and reflective thinking and encourages the development of skills, behaviors and attitudes essential to the good professional practice of future engineers.

Keywords: Active methodologies. Aquarium. Debate. Brainstorming. Group dynamic. 